\title{
Spherical Venn Diagrams with Involutory Isometries
}

\author{
Frank Ruskey ${ }^{\dagger} \quad$ Mark Weston \\ Department of Computer Science \\ University of Victoria \\ PO BOX 3055, Victoria, BC Canada V8W 3P6 \\ \{ruskey, mweston\}@cs.uvic.ca
}

Submitted: Jan 14, 2011; Accepted: Sep 9, 2011; Published: Sep 26, 2011

Mathematics Subject Classification: 68R10

\begin{abstract}
In this paper we give a construction, for any $n$, of an $n$-Venn diagram on the sphere that has antipodal symmetry; that is, the diagram is fixed by the map that takes a point on the sphere to the corresponding antipodal point. Thus, along with certain diagrams due to Anthony Edwards which can be drawn with rotational and reflective symmetry, for any isometry of the sphere that is an involution, there exists an $n$-Venn diagram on the sphere invariant under that involution. Our construction uses a recursively defined chain decomposition of the Boolean lattice.
\end{abstract}

Keywords: Venn diagrams, symmetry, involution, isometry, chain decompositions of the Boolean lattice.

\section{Introduction}

Following Grünbaum [8], an $n$-curve Venn diagram is defined to be a collection of $n$ simple closed curves, $\Theta=\left\{\theta_{1}, \theta_{2}, \ldots, \theta_{n}\right\}$, with the property that for each $S \subseteq\{1,2, \ldots, n\}$ the region

$$
\bigcap_{i \in S} \operatorname{interior}\left(\theta_{i}\right) \cap \bigcap_{i \notin S} \operatorname{exterior}\left(\theta_{i}\right)
$$

is nonempty and connected. In a Venn diagram the curves are assumed to intersect in only finitely many points. An $n$-Venn diagram is one with $n$ curves.

The isometries of the plane that can be exhibited by a finite diagram are subsets of reflections or rotations, and most research on symmetric Venn diagrams has been concerned with rotationally-symmetric diagrams where distinct curves in the diagram map

\footnotetext{
${ }^{\dagger}$ Research supported in part by NSERC.
} 
onto one another. A natural question is: for which $n$ is there a planar $n$-Venn diagram with non-trivial rotational symmetry? It is necessary that $n$ be prime $[10,15]$, and sufficiency was recently proven by Griggs, Killian and Savage (GKS). Our construction is similar to the GKS construction in that it recursively builds the dual graph from a chain decomposition of the Boolean lattice. See the survey [13] for discussion and examples regarding Venn diagrams with rotational symmetry on the plane.

The sphere, on the other hand, has 14 different types of isometry groups, seven of which are infinite classes (see [1]). Grünbaum [9] was the first to discuss Venn diagrams drawn on the sphere, though not in the context of symmetry. It is natural to ask: given an isometry $I$ of the sphere, is there an $n$-Venn diagram on the sphere that is invariant (in some sense) under $I$ ?

In this paper we consider isometries of order two (i.e., the isometry is an involution). Each such isometry is realized by a diagram where each curve gets mapped to itself (what is termed a total symmetry in diagrams to distinguish it from the type where curves get mapped to other curves). The only involutory isometries of the sphere are reflection across a plane containing the centre of the sphere, rotation of the sphere by $\pi$ radians about a diagonal of the sphere, and a rotation of the sphere by $\pi$ about a diagonal $d$ followed by reflection through a plane orthogonal to $d$ through the centre of the sphere (this is called inversion or antipodal symmetry and is equivalent to mapping each point of the sphere to the antipodal point).

The contributions of this paper are: first, to show that the existing Edwards diagrams realize rotational and reflective symmetry, and second, to provide a construction, for any $n$, of an $n$-Venn diagram with antipodal symmetry on the sphere. Thus, for any involutory isometry $f$ of the sphere and any $n>1$, there is an $n$-Venn diagram on the sphere that is invariant under $f$.

\section{Reflective and Rotationally Symmetric Venn Dia- grams on the Sphere}

Anthony Edwards, in a series of papers $[6,5]$, gave a construction, for any $n$, of an $n$ Venn diagram on the plane. Edwards' construction is topologically isomorphic to earlier constructions such as Grünbaum's construction from 1975 [8], but Edwards was the first to note the possibility of observing interesting symmetries of the diagrams produced by embedding them on the sphere, though he did not extend his analysis to all $n$. Edwards' constructions are also discussed in [6] and [13].

On the sphere the Edwards construction has reflective symmetry across two different planes; see Figure 1, in which the planes of symmetry are the two vertical planes containing the curves through the poles. The cylindrical projection of this diagram is shown in Figure 3 .

Edwards also discussed a variant of his construction which he called binary-form; see Figure 2. These diagrams have three different axes of $180^{\circ}$ rotational symmetry; see the cylindrical projection in Figure 4, in which the axes of symmetry are through $x-x^{\prime}$, 
through $y-y^{\prime}$, and through the poles of the sphere.

Thus the Edwards constructions demonstrate that two of the three non-trivial involutions on the sphere are realizable by $n$-Venn diagrams, for any $n$.

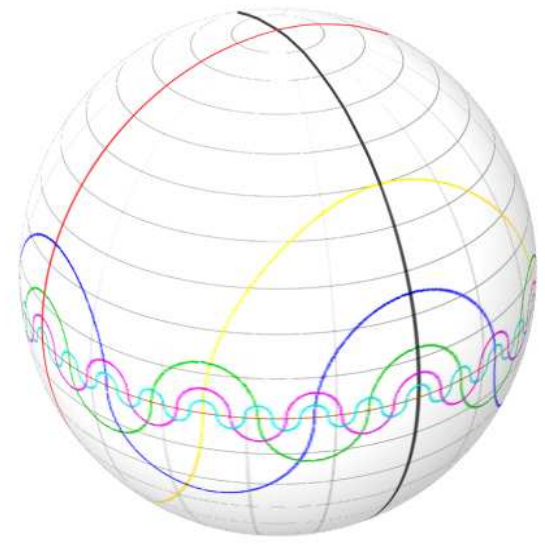

Figure 1: The Edwards construction for a symmetric spherical 6-Venn diagram.

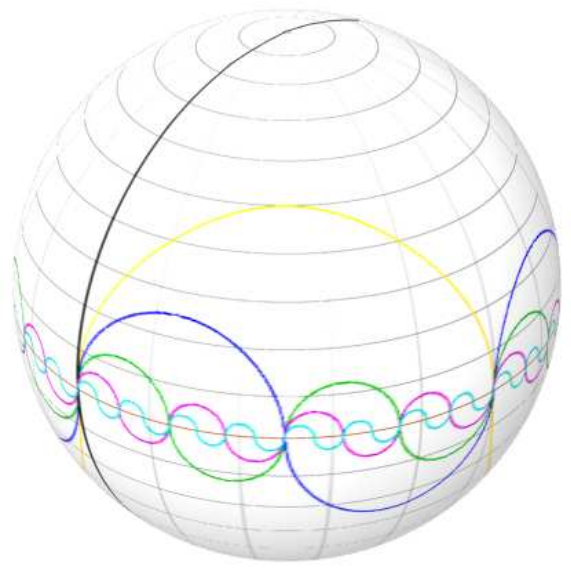

Figure 2: Edwards binary form construction drawn symmetrically on the sphere.

In order to construct $n$-Venn diagrams on the sphere with the third type of symmetry, antipodal symmetry, next we will recursively define the dual graph of such a diagram and show that it has the required symmetry.

\section{The Boolean Lattice and Chain Decompositions}

Let $B_{n}$ be the set of all subsets of $\{1,2, \ldots, n\}$ and let $\mathcal{B}_{n}$ denote the corresponding Boolean lattice. The $2^{n}$ elements of $B_{n}$ correspond naturally to the bitstrings of length $n$ where a bit in position $i$ in a bitstring is 1 if and only if the corresponding subset includes element $i$. The cover relations of $\mathcal{B}_{n}$ are then exactly those pairs of bitstrings $a \subset b$ such that $a$ and $b$ differ in one bit position where $a$ has a 0 and $b$ has a 1 . In $\mathcal{B}_{n}$ elements are ranked by the Hamming weight of the corresponding bitstring (the size of the subset).

An elegant chain decomposition of the Boolean lattice $\mathcal{B}_{n}$ was given by de Bruijn, et al. in 1951 [2], and studied by many subsequent authors. In the important paper by Griggs, Killian and Savage [7], it is shown how de Bruijn's symmetric chain decomposition of $\mathcal{B}_{n}$ can be used to construct $n$-Venn diagrams. They then create a new symmetric chain decomposition with a certain $n$-fold symmetry and use it to create $n$-fold rotationally symmetric $n$-Venn diagrams. This in turn has seemingly spawned recent renewed interest in symmetric chain decompositions with additional symmetries by several authors $[3,4$, 11]. 


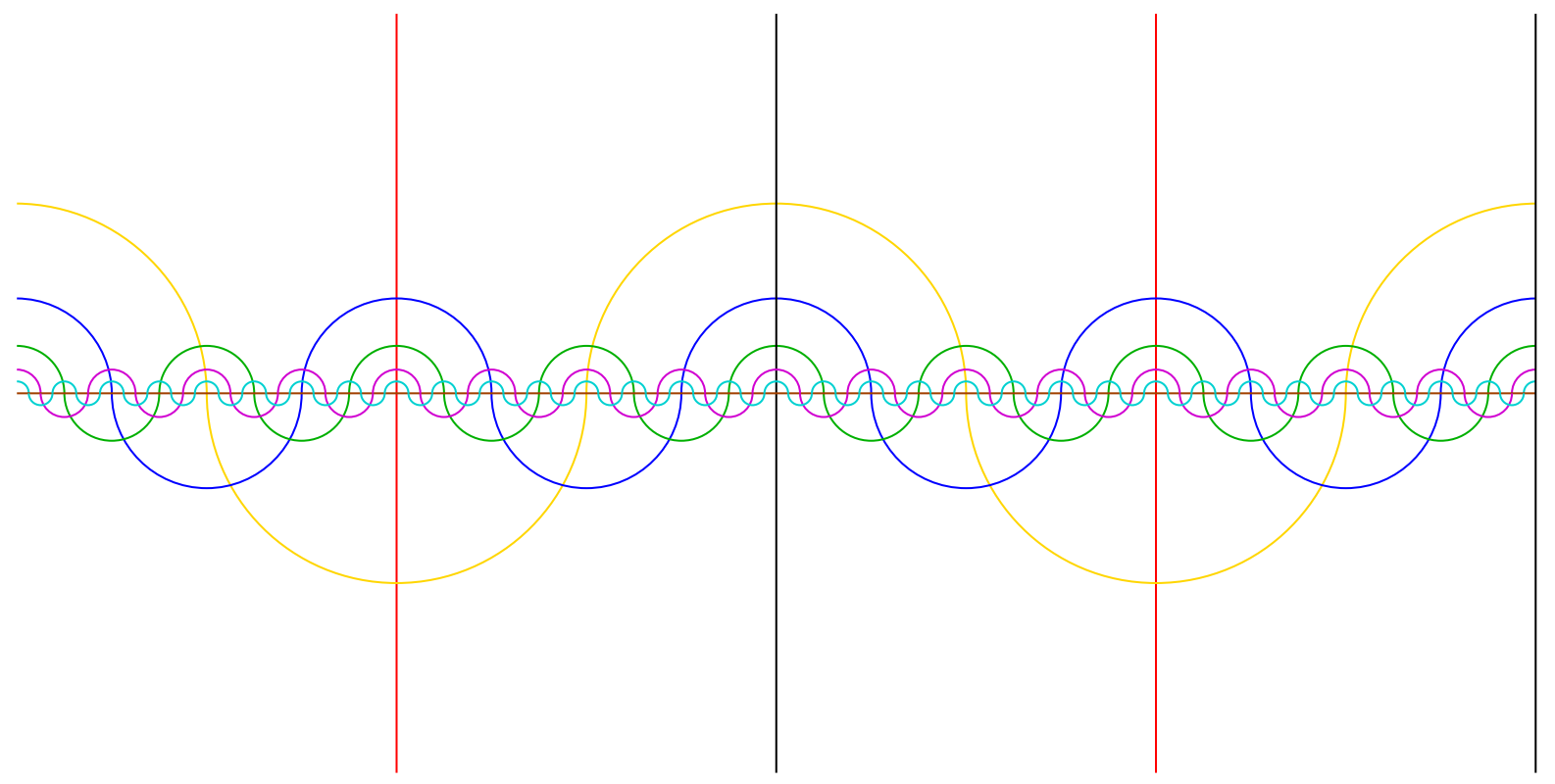

Figure 3: Cylindrical projection of the Edwards construction for an 6-Venn diagram.

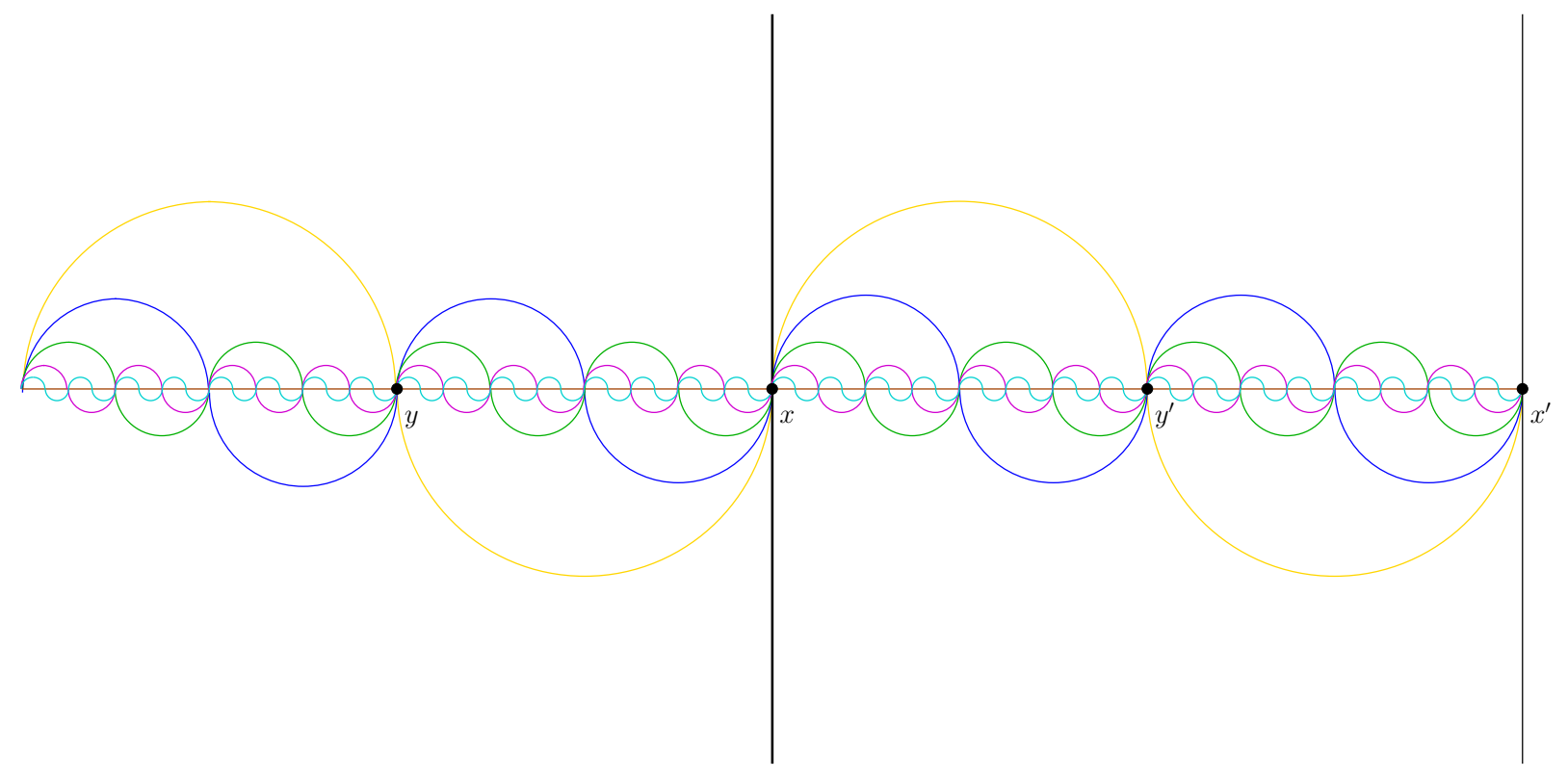

Figure 4: Cylindrical projection of Edwards binary-form construction. 


\subsection{Embeddings of Chain Decompositions}

The embeddings of some chain decompositions of $\mathcal{B}_{n}$ have the property that they form a spanning subgraph of a dual graph of a Venn diagram. If $C$ is a chain decomposition then we define the following notation.

$$
\max (C):=\left\{v \in B_{n} \mid v \text { is maximal in some chain of } C\right\}
$$

and

$$
\min (C):=\left\{v \in B_{n} \mid v \text { is minimal in some chain of } C\right\},
$$

Definition. Let $E$ be a planar embedding of an ordered chain decomposition $C=$ $\left\{C_{0}, C_{1}, \ldots, C_{m-1}\right\}$ in which each chain forms a vertical line segment, and the chains are indexed so that $C_{i}$ is to the left of $C_{j}$ if $i<j$. Then $E$ is said to be a venerable embedding if there are functions $\pi: \max (C) \backslash\left\{1^{n}\right\} \rightarrow \max (C)$ and $\pi^{\prime}: \min (C) \backslash\left\{0^{n}\right\} \rightarrow \min (C)$ such that the edge sets $E_{\pi}=\left\{\{v, \pi(v)\} \in \mathcal{B}_{n} \mid v \in \max (C) \backslash\left\{1^{n}\right\}\right\}$ and $E_{\pi^{\prime}}=\left\{\left\{v, \pi^{\prime}(v)\right\} \in\right.$ $\left.\mathcal{B}_{n} \mid v \in \min (C) \backslash\left\{0^{n}\right\}\right\}$ can be added to $E$ without creating any crossing edges; the edges in $E_{\pi} \cup E_{\pi^{\prime}}$ are called chain cover edges. Note: in some of the later sections, we draw the chains horizontally instead of vertically, whatever is most convenient.

Note that the two sets of chain cover edges form trees. It was proven in [7] that every venerable embedding is the dual of a Venn diagram (although they use different terminology). Figure 5 shows an example of a venerable embedding in which the thin (black) segments indicate chains and the thick (red) segments indicate chain cover edges. Given a bitstring $x$ we denote by $\bar{x}$ the bitstring obtained by complementing each bit of $x$. Also, if $C_{i}$ is a chain then $\bar{C}_{i}$ denotes the chain obtained by complementing each bitstring in $C$ (and the ordering of the chain elements is reversed).

Definition. Let $E$ be a venerable embedding of an ordered chain decomposition $C=$ $\left\{C_{0}, C_{1}, \ldots, C_{m-1}\right\}$. The embedding $E$ is an antipodally symmetric chain decomposition embedding (or is an $A S C D E$ ) if the following conditions are met.

(a) $C_{i}=\bar{C}_{i+m / 2}$ for $i=0,1, \ldots, m-1$, with indexing taken $\bmod m$.

(b) If $(v, \pi(v)) \in E_{\pi}$, then $\left(\bar{v}, \overline{\pi^{\prime}(v)}\right) \in E_{\pi^{\prime}}$.

In Section 4, we will show how to construct antipodally symmetric chain decomposition and then in Section 5 how to use such an embedding to create a Venn diagram on the sphere with antipodal symmetry.

\section{Constructing an Antipodally Symmetric Chain De- composition}

In this section we develop a variation of the chain decomposition decomposition of de Bruijn, van Ebbenhorst Tengbergen, and Kruyswijk [2] with the chain ordering of Knuth [12, pg. 457], and use it to to create an antipodally symmetric chain decomposition. 
We define the construction recursively, along with an embedding on the plane, following Knuth [12]'s presentation of the de Bruijn decomposition, and specify chain cover edges as well as the chains for each step in the construction.

Lemma 4.1. Given an $A S C D E M$ for $\mathcal{B}_{n}$, there is an $A S C D E M^{\prime}$ for $\mathcal{B}_{n+1}$.

Proof. The following recursive rules allow us to construct, given a ASCDE $M$ for $\mathcal{B}_{n}$, a ASCDE $M^{\prime}$ for $\mathcal{B}_{(n+1)}$.

Let $m$ be the number of chains in $M$. The $i$ th chain has chain cover edges embedded as shown below. We refer to this configuration as the $i$-th row. The chain cover edges are the thick (red) edges that connect different chains together; cover edges in the chains are thin (black).

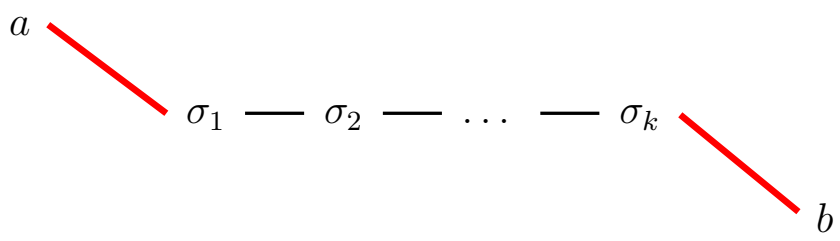

The row is replaced in one of two ways, depending on whether it is in the first half of the $\operatorname{ASCDE}\left(i . e\right.$. whether $\left.i \leq \frac{m}{2}\right)$.

Rule 1. If $i \leq \frac{m}{2}$, then the row is replaced by the following two rows and chain cover edges.

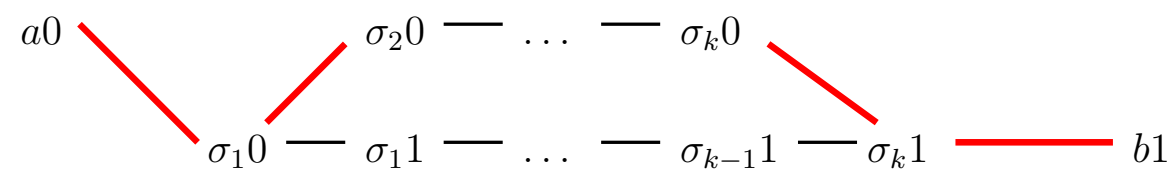

If $k=1$ the first row is omitted and the second becomes $\sigma_{1} 0-\sigma_{1} 1$. Note that two new chain cover edges are added: between $\alpha_{1} 0$ and $\alpha_{2} 0$, and between $\alpha_{k} 0$ and $\alpha_{k} 1$.

Rule 2. If $i>\frac{m}{2}$, the replacement is shown below.

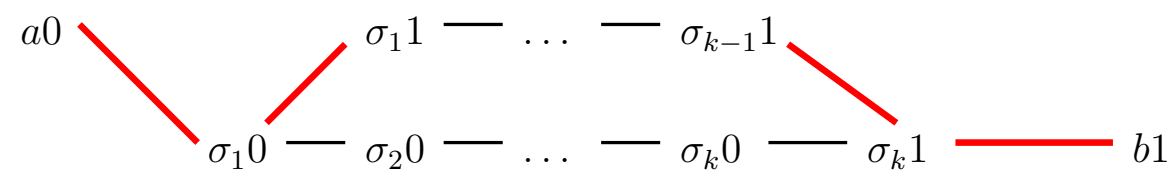

If $k=1$ the first row is omitted and the second becomes $\sigma_{k} 0-\sigma_{k} 1$. Note again that two new chain cover edges are added: between $\alpha_{1} 0$ and $\alpha_{1} 1$, and between $\alpha_{k-1} 1$ and $\alpha_{k} 1$. 
Replacing every row in $M$ in this fashion gives $M^{\prime}$; we must show that $M^{\prime}$ is an ASCDE. It is easy to show by induction that each of the $2^{n+1}$ bitstrings appears exactly once, the bit strings with $k$ s all appear in the same column, and within each row, consecutive bit strings differ by changing a 0 to a 1 . Thus $M^{\prime}$ does give a chain decomposition.

Given a chain $C_{i}=\sigma_{1} \sigma_{2} \ldots \sigma_{k}$ in the first $m / 2$ rows of $M$, since $M$ is a ASCDE, we have $C_{i+m}=\overline{\sigma_{k}} \ldots \overline{\sigma_{2}} \overline{\sigma_{1}}$. Rule 1 creates chains

$$
C_{i}^{\prime}=\sigma_{2} 0, \ldots, \sigma_{k} 0 \text { and } C_{i}^{\prime \prime}=\sigma_{1} 0, \sigma_{1} 1, \sigma_{2} \ldots, \sigma_{k} 1 .
$$

Rule 2 creates the corresponding chain

$$
C_{i+m}^{\prime}=\overline{\sigma_{k}} 1, \ldots, \overline{\sigma_{2}} 1 \text { and } C_{i+m}^{\prime \prime}=\overline{\sigma_{k}} 0, \ldots, \overline{\sigma_{2}} 0, \overline{\sigma_{1}} 1 .
$$

Since $C_{i}^{\prime}=\overline{C_{i+m}^{\prime}}$ and $C_{i}^{\prime \prime}=\overline{C_{i+m}^{\prime \prime}}$ condition (a) of the ASCDE definition is satisfied. Condition (b) is satisfied since the old chain cover edges are preserved in the sense that $\left(\sigma_{1}, a\right)$ and $\left(\sigma_{k}, b\right)$ become $\left(\sigma_{1} 0, a 0\right)$ and $\left(\sigma_{k} 1, b 1\right)$; furthermore, the new chain cover edges come in pairs $\left(\sigma_{2} 0, \sigma_{1} 0\right)$ and $\left(\overline{\sigma_{2}} 1, \overline{\sigma_{1}} 1\right)$.

Theorem 4.2. There exists an antipodally symmetric chain decomposition embedding $(A S C D E)$ of $\mathcal{B}_{n}$ for all $n \geq 2$.

Proof. The basis is an ASCDE for $\mathcal{B}_{2}$, shown below. Now apply Lemma 4.1.

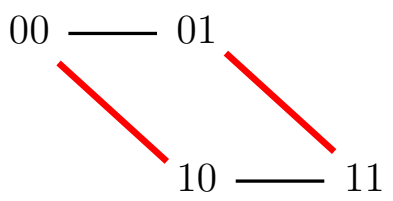

In general, there are many ASCDEs for a given $n$; the construction of Lemma 4.1, starting from the base case shown for $n=2$, gives us just one of them. Figure 5 shows the construction for $n=4$ and the background (shown in grey) in Figure 6 shows the construction for $n=5$.

\section{Spherical Venn Diagrams with Antipodal Symme- try}

In this section we explain why an ASCDE can be drawn on the sphere with antipodal symmetry and how the dual can then be drawn as a Venn diagram on the sphere, also with antipodal symmetry. Given a cylindrical projection of a sphere to a rectangle, imagine dividing the rectangle into two equal area smaller rectangles by bisecting the rectangle with a vertical line. A fundamental region for antipodal symmetry is obtained by taking a smaller rectangle, say the one to the left, call it $L$. The antipodal mapping, call it $\Xi$, takes $L$, flips it vertically, and places it to the right of $L$. 


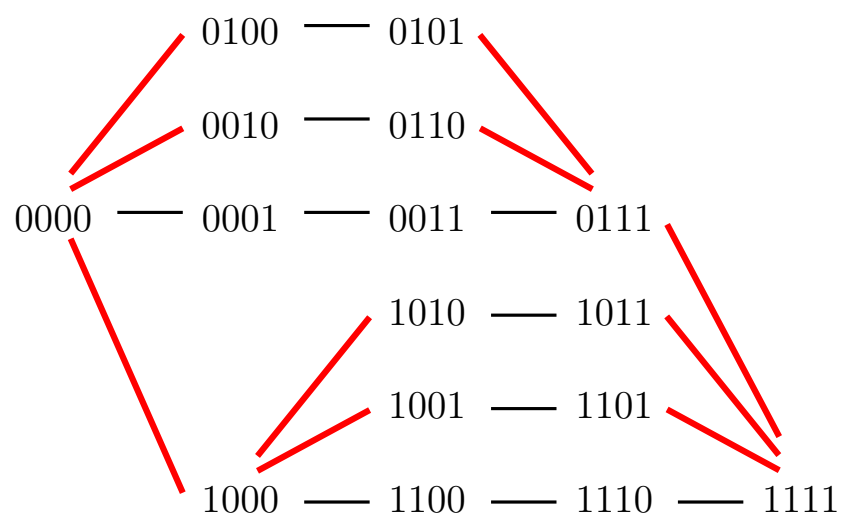

Figure 5: The ASCDE for $\mathcal{B}_{4}$ generated by the recursive construction

Given an ASCDE $E$ with $m$ ordered chains, embed the first $m$ chains in $L$. Using $\Xi$ to position the remaining chains, the embedding now has the property that any bitstring $\sigma$ is antipodal to $\bar{\sigma}$ (this is because of the condition $C_{i}=\bar{C}_{i+m}$ in the definition of an ASCDE). The background (shown in grey) in Figure 6 illustrates such an embedding.

The dual of a (primal) graph embedded on a surface can be drawn so as to preserve the symmetries of the embedding of the primal; thus, the dual of $E$ can be drawn so that it has antipodal symmetry. Since $E$ is venerable, there is an $n$-Venn diagram on the sphere with antipodal symmetry corresponding to the cylindrical projection. Figure 6 illustrates an 5-Venn diagram with antipodal symmetry constructed from our ASCDE and Figure 7 shows the spherical version. The preceding discussion is summarized by the following theorem.

Theorem 5.1. For any involutory isometry of the sphere $f$ and $n \geq 1$, there is a spherical $n$-Venn diagram that is fixed by $f$.

\section{$6 \quad$ Further Remarks}

\subsection{Rotationally Symmetric Chain Decompositions and Dia- grams}

Consider the effect of changing Rule 2 to the following:

Rule $2^{\prime}$. If $i>\frac{m}{2}$, the row becomes

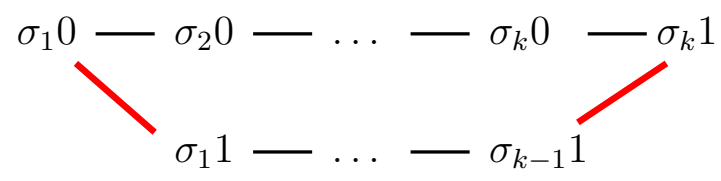




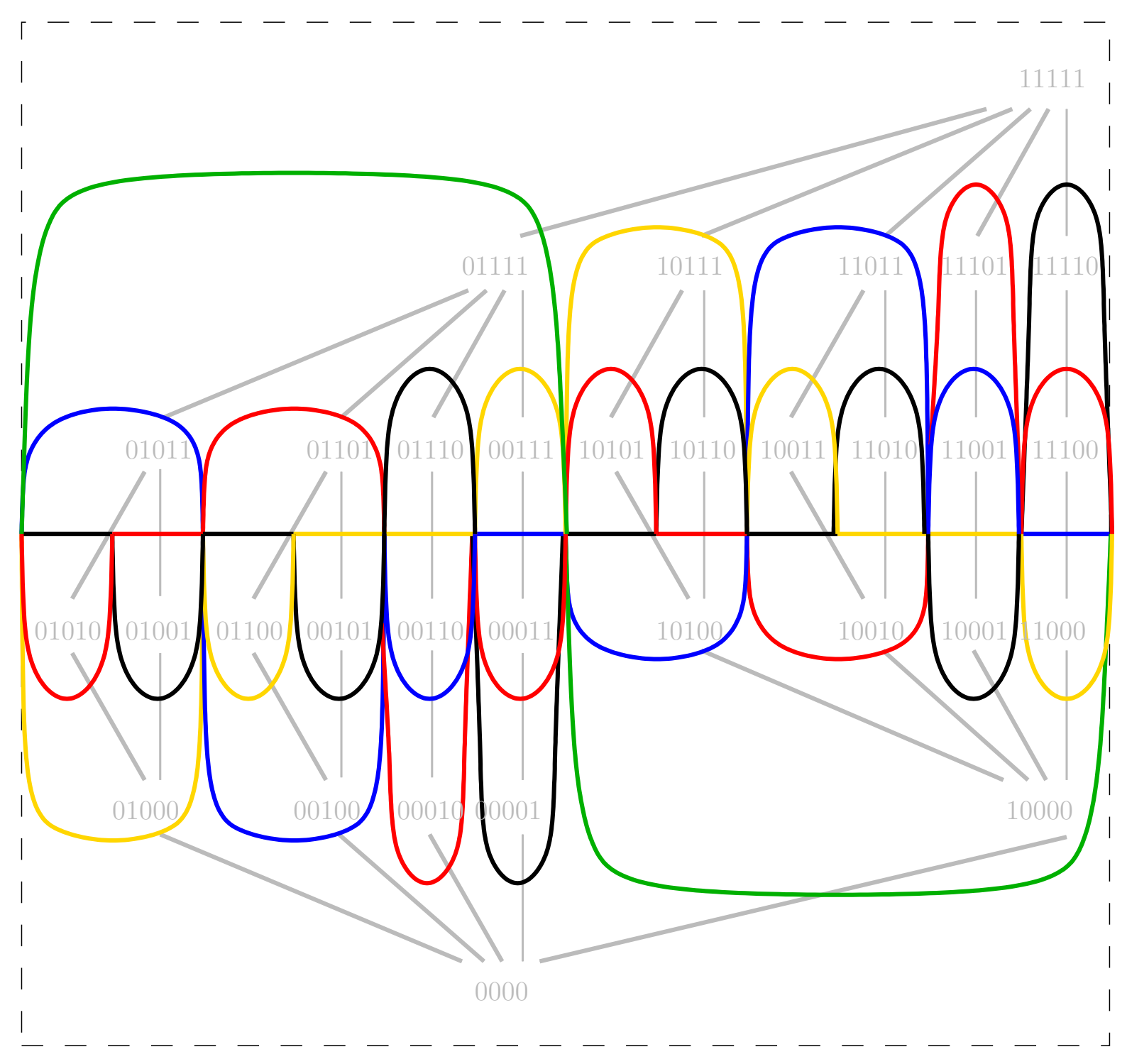

Figure 6: Antipodally symmetric 5-curve diagram based on the ASCDE for $\mathcal{B}_{5}$

where if $k=1$ the second row is omitted and the first becomes $\sigma_{k} 0-\sigma_{k} 1$.

That is, the order of the two rows is reversed, which has the effect of reversing the order of the chains in the second half of the construction. Then the chain decomposition has a different symmetry: the first chain $C_{0}$ maps onto $C_{m-1}, C_{2}$ onto $C_{m-2}$, etc. until $C_{m / 2-1}$ which maps onto $C_{m / 2}$. Once the projection onto the sphere is complete, the resulting diagrams do not have antipodal symmetry but rather a rotational symmetry about an axis passing through the centre of the sphere and the central vertex between $C_{m / 2}$ and $C_{m / 2+1}$. Thus, for any $n$, we have a chain-based construction for an $n$-Venn diagram on the sphere with total rotational symmetry, as opposed to the Edwards diagram shown 


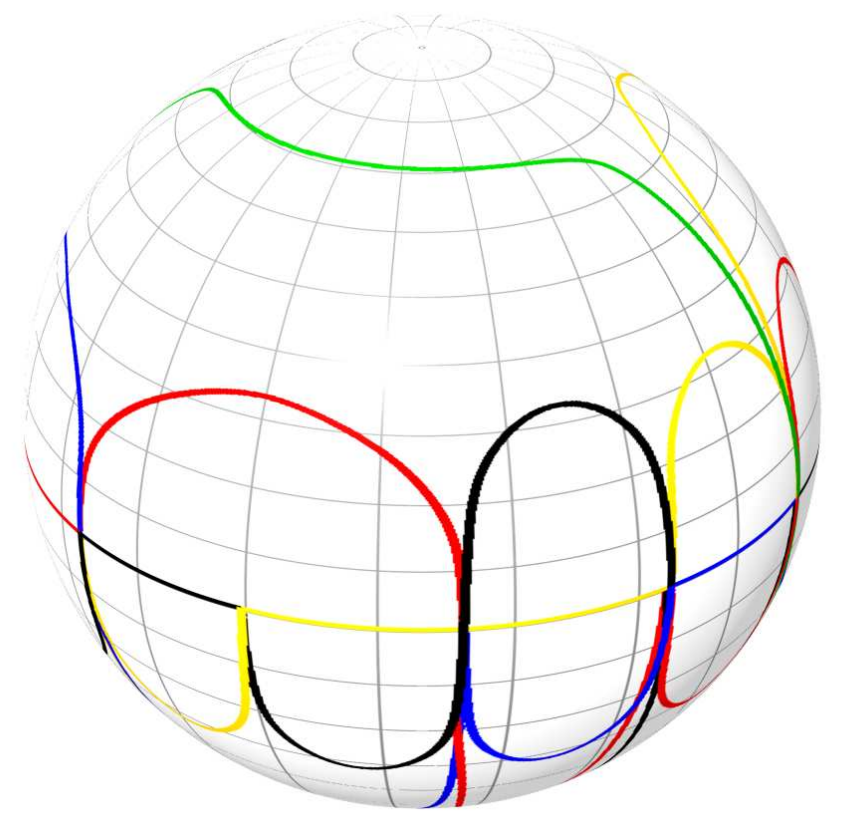

Figure 7: Antipodally symmetric 5-Venn diagram from the ASCDE for $\mathcal{B}_{5}$ on the sphere

earlier in this paper, which has a diagrammatic construction. The Edwards construction results in $2^{n-1}$ chains, for $n$ curves, whereas our construction has fewer chains, as we see in the next section. The resulting Venn diagrams are explored further in [16].

\subsection{Counting Results}

In this section we count the number of chains in the ASCDE construction. The solution involves the Catalan numbers $\mathbf{C}_{n}=\left(\begin{array}{c}2 n \\ n\end{array}\right) /(n+1)=\left(\begin{array}{c}2 n+1 \\ n\end{array}\right) /(2 n+1)$. Let $N(n, k)$ be the number of chains of length $k$ in the construction. Also, define the total number of chains $N(n)=\Sigma_{1 \leq k \leq n} N(n, k)$. Directly from the recursive construction, we have the recurrence relation

$$
N(n, k)= \begin{cases}2 & \text { if } n=k \geq 1, \\ N(n-1,2) & \text { if } k=1, \\ N(n-1, k-1)+N(n-1, k+1) & \text { if } k>1, \\ 0 & \text { otherwise. }\end{cases}
$$

Lemma 6.1. If $n$ and $k$ have the same parity, then

$$
N(n, k)=\frac{2 k}{n}\left(\begin{array}{c}
n \\
(n-k) / 2
\end{array}\right)
$$

and, otherwise, $N(n, k)=0$. 
Proof. The recurrence relation (2) is the same as one for the Catalan triangle [14, Sequence A053121], except that the base case is 2 instead of 1 . The reference [14, Sequence A053121] gives the closed form $\frac{k}{n}\left(\begin{array}{c}n \\ (n-k) / 2\end{array}\right)$ for the Catalan triangle (also easily verified by induction), and twice this is the solution to (2).

Corollary 6.2. If $n=2 m+1$ is odd, then $N(n, 1)=2 \mathbf{C}_{m}$; otherwise $N(n, 1)=0$.

Proof. If $n$ is odd, let $m=2 n+1$. Then

$$
N(n, 1)=\frac{2}{n}\left(\begin{array}{c}
n \\
(n-1) / 2
\end{array}\right)=\frac{2}{2 m+1}\left(\begin{array}{c}
2 m+1 \\
m
\end{array}\right)=2 \mathbf{C}_{m} .
$$

If $n$ is even, the parity of $n$ and $k$ differ, so by Lemma $6.1, N(n, 1)=0$.

Lemma 6.3. If $n$ is even, all chains in the chain decomposition given by the ASCDE construction have even length, otherwise all chains have odd length.

Proof. The lemma is easily verified to be true for $n=2$ and $n=3$. Assume the lemma is true for $n-1$ and $n-2$. Let $n$ be even and assume the construction for $n-1$ produces chains of only odd lengths. The rule replaces a chain of odd length $k>1$ with two chains of length $k+1$ and $k-1$, so their lengths must both be even; the rule produces one chain of length two if $k=1$. In both cases the resulting chains all have even length. The case for $n$ odd is similar and is omitted.

Theorem 6.4. The number of chains $N(n)$ in the ASCDE construction for $n>1$ is $\left(\begin{array}{c}n \\ n / 2\end{array}\right)$ if $n$ is even, and is $2\left(\begin{array}{c}n-1 \\ (n-1) / 2\end{array}\right)$ otherwise.

Proof. Our proof is by induction on $n$. The basis for $n=2$ is shown in Theorem 4.2 and the basis for $n=3$ is easily verified. We now consider two cases, $n$ odd and even.

For $n$ odd, inductively the number of chains in the construction for $n-1$ is $\left(\begin{array}{c}n-1 \\ (n-1) / 2\end{array}\right)$. By Lemma 6.3 all chains in the construction for $n-1$ are of even length and so are all replaced by two odd chains; from the construction it is apparent that each even-length chain gives exactly two odd-length chains. Thus for $n$ odd, $N(n)=2\left(\begin{array}{c}n-1 \\ (n-1) / 2\end{array}\right)$.

For $n$ even, the construction for $n-1$ gives $2\left(\begin{array}{c}n-2 \\ (n-2) / 2\end{array}\right)$ chains by induction, and all chains have odd length. Applying the construction rule gives two chains for every chain in the construction for $n-1$, except for chains of length one which will give one chain. By Lemma 6.2 the number of length one chains is

$$
N(n-1,1)=2 \mathbf{C}_{\frac{n-2}{2}}=\frac{2}{(n-2) / 2+1}\left(\begin{array}{c}
n-2 \\
(n-2) / 2
\end{array}\right)=\frac{4}{n}\left(\begin{array}{c}
n-2 \\
(n-2) / 2
\end{array}\right) .
$$


Thus the number of chains for $n$ even is

$$
\begin{aligned}
N(n) & =2 N(n-1)-N(n-1,1) \\
& =2 \cdot 2\left(\begin{array}{c}
n-2 \\
(n-2) / 2
\end{array}\right)-\frac{4}{n}\left(\begin{array}{c}
n-2 \\
(n-2) / 2
\end{array}\right) \\
& =4 \frac{n-1}{n}\left(\begin{array}{c}
n-2 \\
(n-2) / 2
\end{array}\right) \\
& =\left(\begin{array}{c}
n \\
n / 2
\end{array}\right), \text { as claimed. }
\end{aligned}
$$

It is well-known that the minimum possible number of chains in a chain decomposition

is $\left(\begin{array}{c}n \\ \lfloor n / 2\rfloor\end{array}\right)$. The results above show that the construction may, depending on $n$, give a chain decomposition with more than the minimum number of chains possible. An easy computation reveals that when $n$ is odd, the difference is

$$
N(n)-\left(\begin{array}{c}
n \\
(n-1) / 2
\end{array}\right)=\mathbf{C}_{\frac{n-1}{2}} .
$$

\subsection{Open Questions}

We noted in the previous section that the construction gives more than the minimum number of chains, starting with $n \geq 5$. The ASCDE shown in Figure 8 for $n=5$ contains $\left(\begin{array}{c}5 \\ \lfloor 5 / 2\rfloor\end{array}\right)=10$ chains, as opposed to the number $N(5)=12$, from Subsection 6.2, given by the construction.

We conjecture that there exists an ASCDE with $\left(\begin{array}{c}n \\ (n-1) / 2\end{array}\right)$ chains whenever $n+1$ is not a power of 2 . If $n+1$ is a power of 2 , then the middle binomial coefficient is odd and thus there is no antipodally symmetric decomposition with this number of chains.

\section{Acknowledgements}

We wish to thank an anonymous referee for some helpful suggestions and references.

\section{References}

[1] John H. Conway, Heidi Burgiel, and Chaim Goodman-Strass. The Symmetries of Things. A K Peters, 2008.

[2] N. G. de Bruijn, Ca. van Ebbenhorst Tengbergen, and D. Kruyswijk. On the set of divisors of a number. Nieuw Arch. Wiskunde (2), 23:191-193, 1951.

[3] Vivek Dhand. Symmetric chain decomposition of necklace posets. http://arxiv.org/abs/1104.4147, 2011. [Online; Arxiv e-print]. 


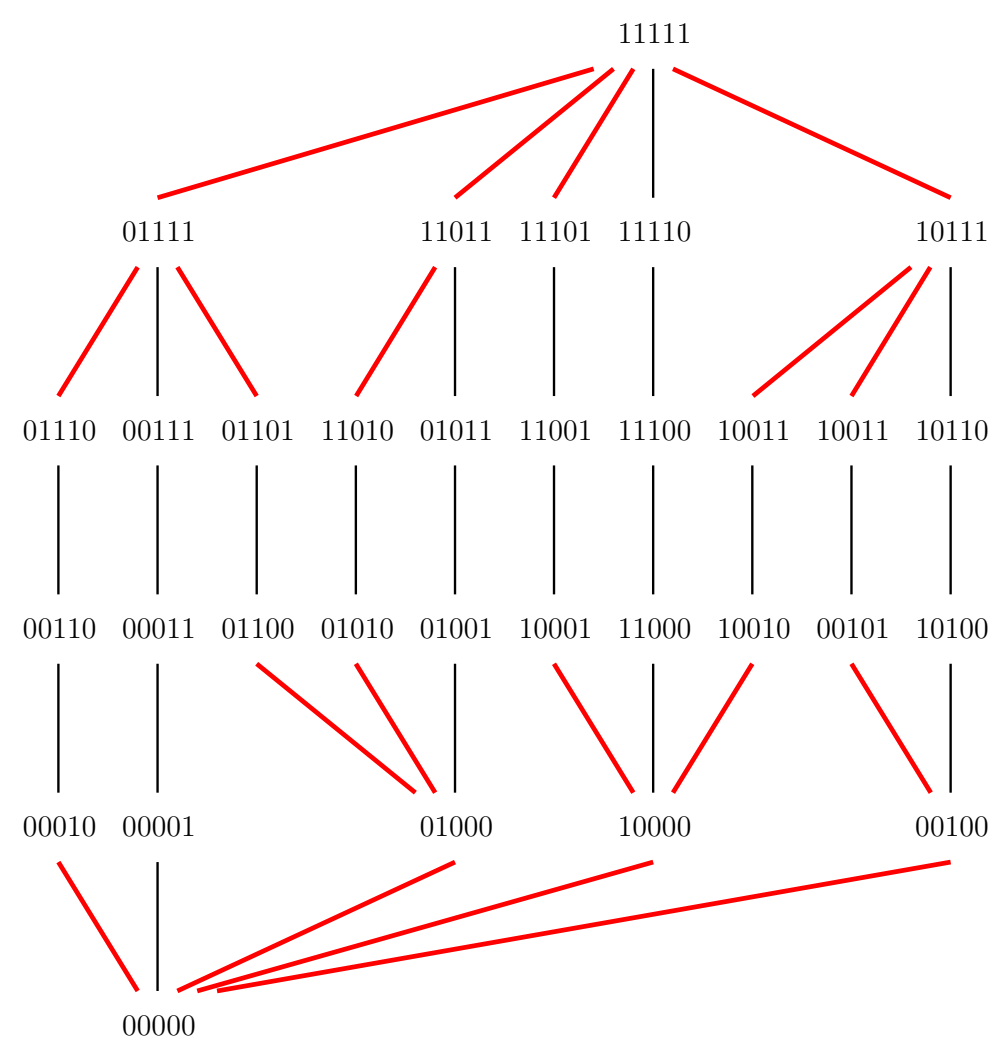

Figure 8: $\mathrm{ASCDE}$ of $\mathcal{B}_{5}$ with minimum number of chains

[4] Dwight Duffus, Jeremy McKibben-Sanders, and Kyle Thayer. Some quotients of the Boolean lattice are symmetric chain orders. http://arxiv.org/abs/1107.1098, 2011. [Online; Arxiv e-print].

[5] A. W. F. Edwards. Venn diagrams for many sets. New Scientist, 7:51-56, Jan 1989.

[6] A. W. F. Edwards. Cogwheels of the Mind: The Story of Venn Diagrams. John Hopkins University Press, 2004.

[7] J. Griggs, C. E. Killian, and C. Savage. Venn diagrams and symmetric chain decompositions in the boolean lattice. The Electronic Journal of Combinatorics, 11(1), 2004. Article R2 (online).

[8] B. Grünbaum. Venn diagrams and independent families of sets. Mathematics Magazine, 48:12-23, 1975.

[9] B. Grünbaum. Venn diagrams I. Geombinatorics, 1:5-12, 1992.

[10] D. Henderson. Venn diagrams for more than four classes. American Mathematical Monthly, 70(4):424-426, 1963.

[11] Patricia Hersh and Anne Schilling. Symmetric chain decomposition for cyclic quotients of Boolean algebras and relation to cyclic crystals. http://arxiv.org/abs/1107.4073, 2011. [Online; Arxiv e-print]. 
[12] Donald Knuth. The Art of Computer Programming: Vol. 4A: Combinatorial Algorithms Part 1. Pearson Education, Boston, 2011.

[13] F. Ruskey and M. Weston. A survey of Venn diagrams. The Electronic Journal of Combinatorics, 1997. Dynamic survey, Article DS5 (online). Revised 2001, 2005.

[14] N. J. A. Sloane. The On-Line Encyclopedia of Integer Sequences. http://www.research.att.com/ njas/sequences/, 2007. [Online; accessed 8-April-2008].

[15] Stan Wagon and Peter Webb. Notes: Venn symmetry and prime numbers: a seductive proof revisited. American Mathematical Monthly, August/September 2008.

[16] M. Weston. Symmetric Venn diagrams on the sphere. PhD thesis, University of Victoria, 2009. 